\title{
In situ chemically specific mapping of agrochemical seed coatings using stimulated Raman scattering microscopy
}

\author{
Chun-Chin Wang ${ }^{1}$, Siân Moorhouse ${ }^{2}$, Chris Stain ${ }^{2}$, Mark Seymour ${ }^{2}$, Ellen Green ${ }^{1}$, \\ Steven Penfield ${ }^{3}$, and Julian Moger ${ }^{1, *}$ \\ ${ }^{1}$ School of Physics, University of Exeter, Exeter, EX4 4QL, United Kingdom; \\ ${ }^{2}$ Syngenta, Jealott's Hill International Research Centre, Bracknell, RG42 6EY, United \\ Kingdom; \\ ${ }^{3}$ Department of Crop Genetics, John Innes Centre, Norwich Research Park, Norwich, \\ NR4 7UH, United Kingdom \\ *Corresponding Author: E-mail: J.Moger@exeter.ac.uk
}

\begin{abstract}
Providing sufficient, healthy food for the increasing global population is putting a great deal of pressure on the agrochemical industry to maximise crop yields without sustaining environmental damage. The growth and yield of every plant with sexual reproduction, depends on germination and emergence of sown seeds, which is affected greatly by seed disease. This can be most effectively controlled by treating seeds with pesticides before they are sown. An effective seed coating treatment requires a high surface coverage and adhesion of active ingredients onto the seed surface and the addition of adhesive agents in coating formulations plays a key role in achieving this. Although adhesive agents are known to enhance seed germination, little is understood about how they affect surface distribution of actives and how formulations can be manipulated to rationally engineer seed coating preparations with optimized coverage and efficacy. We show, for the first time, that stimulated Raman scattering (SRS) microscopy can be used to map the seed surface with microscopic spatial resolution and with chemical specificity to identify formulation components distributed on the seed surface. This represents a major advance in our capability to rationally engineer seed coating formulations with enhanced efficacy.
\end{abstract}

\section{Keywords:}

Label-free, stimulated Raman scattering, epi-SRS, fungicide, wheat, seed coating

This article has been accepted for publication and undergone full peer review but has not been through the copyediting, typesetting, pagination and proofreading process, which may lead to differences between this version and the Version of Record. Please cite this article as doi: 10.1002/jbio.201800108 


\section{INTRODUCTION}

The rapidly increasing global population and growing demand for improved diet in the developing world is putting ever increasing pressure on the agricultural and agrochemical industries to enhance agricultural yields. Seed germination and seedling establishment are key factors in crop yield and both are heavily affected by seed disease. An effective strategy to control seed disease and hence increase seed performance is to treat seeds before sowing by coating them with active agents to control moisture, plant growth, pest damage and disease ${ }^{[1-4]}$. With this approach active ingredients (Als) are applied specifically to target the seed during germination to maximise their performance whilst at the same time minimising human exposure, environmental pollution and costs. An effective seed coating treatment requires a high surface coverage and adhesion of actives onto the seed surface and the addition of adhesive agents in seed coating formulations plays a key role in achieving this.

The ability to map the seed surface with microscopic spatial resolution and with chemical specificity to identify formulation components distributed on the seed surface would represent a major advance in our capability to rationally engineer seed coating formulations with enhanced active coverage. However, conventional analytical tools fall short of this requirement. Various methods, including HPLC-mass spectrometry (HPLC-MS) measurement ${ }^{[5]}$, Raman spectroscopy ${ }^{[6,7]}$, X-ray Micro-CT ${ }^{[8]}$, and near infrared hyperspectral spectroscopy ${ }^{[9]}$ have been utilized to analyse the distribution of agrochemicals on the seed surfaces. However, these techniques suffer from a number of drawbacks such as lengthy sample preparation of seed sections ${ }^{[5}$, ${ }^{6]}$, loss of spatial resolution, long acquisition times ${ }^{[7]}$ or lack of chemical specificity for the Als ${ }^{[8,9]}$. Labelling of the Al molecules with fluorophores has also been used to evaluate seed coatings ${ }^{[10]}$, however the low molecular weight of most Als means that fluorescent derivatization has a direct impact on their chemical-physical properties which makes interpretation of the results difficult and potentially misleading.

We demonstrate that a novel label-free imaging technique, stimulated Raman scattering, can overcome the limitations of conventional methods and for the first time provide in situ quantitative analysis which demonstrates how the microscopic distribution of fungicides on wheat seeds can be modified by formulation additives. Using fludioxonil, a non-systemic fungicide, as an example Al, we show SRS can be used to visualise the distribution of the compound on the seed surface and we 
investigate the effect of the addition of vegetable oil, a common co-formulant material, on the distribution of Al on the seed surface.

Stimulated Raman Scattering (SRS) microscopy has emerged as a powerful tool for mapping the spatial-temporal distribution of unlabelled compounds based on vibrational spectroscopy ${ }^{[11-13]}$. SRS generates a coherent non-linear Raman signal by focussing a pump and Stokes laser pulses into a sample with a difference in frequency $\left(\omega_{p}-\omega_{s}\right)$ matched to a Raman active mode of a molecular species of interest. Under this resonant condition the Stokes beam intensity experiences a gain (stimulated Raman gain - SRG) while the pump beam experiences a loss (SRL). The intensity transfer from the pump to the Stokes beam only occurs when both beams are incident upon the sample and can be detected with high sensitivity using modulation transfer detection ${ }^{[11,12]}$. With significant improvement of imaging speed and detection sensitivity ${ }^{[14-16]}$, SRS microscopy has been demonstrated to allow non-invasive imaging of compounds in living cells and tissues ${ }^{[17-22]}$. Moreover, since SRS is detected as a modulation in the excitation fields, it is not susceptible to autofluorescence and has recently been demonstrated to be suitable for application in planta ${ }^{[23-31]}$.

\section{MATERIALS AND METHODS}

\section{Stimulated Raman Scattering Microscopy}

SRS microscopy was performed using a custom-built imaging system based on a commercial laser scanning microscope and a picosecond laser. The laser source consisted of a picosecond mode-locked fibre laser (aeroPULSE, NKT Photonics) providing 2 ps pulses at $1032 \mathrm{~nm}$ which were frequency-doubled to pump an optical parametric oscillator (OPO) (Levante Emerald, APE) ${ }^{[21]}$, which provide a tuneable signal beam tuneable from 690 to $990 \mathrm{~nm}$ which served as the Stokes beam. In the optical setup shown in Figure 1a, pump and Stokes beams with frequencies $\omega_{p}$ and $\omega_{s}$, respectively, are incident upon the sample with the frequency difference $\omega_{p}-\omega_{s}$ chosen to match the molecular vibrational frequency $(\Omega)$ of interest as illustrated in the energy diagram.

The Stokes beam was modulated at $8.25 \mathrm{MHz}$ by using an acoustic optical modulator (AOMO 3080-122, Crystal Technology) and 80 MHz driver (AODR 1080AF-A1F0-1.0, Crystal Technology). All imaging was carried out on a modified confocal laser scan 
unit (Fluoview 300, Olympus) and an inverted microscope (IX71, Olympus). The light was focused onto the sample using a 60× NA1.2 water immersion microscope objective (UPlanSApo, Olympus). The pump and Stokes laser powers on the sample were 6 and $12 \mathrm{~mW}$, respectively. The forward propagated light was collected using a 100× NA1.4 oil immersion microscope objective (UPlanSApo, Olympus), and SRS was detected in the forward direction using a Si photodiode (FDS 1010, Thorlabs) with a $70 \mathrm{~V}$ reverse bias. A schematic of the optical setup is shown in Figure 1a. To collect the back-scattered light from the wheat seed, we installed a quarter-wave plate (AQWP10M-980, Thorlabs) and polarizing beam splitter (CCM1-PBS252, Thorlabs) in the epi-direction, where the photons of the pump beam were detected by a biased $\mathrm{Si}$ photodiode (DET36A, Thorlabs). The schematic illustration of epi-SRS setup is shown in Figure 3a.

For stimulated Raman loss (SRL) imaging, the Stokes beam was blocked with two band-pass filters (CARS $890 / 220 \mathrm{~nm}$, Chroma Technologies) and the pump beam was detected. To suppress the strong signal due to the laser pulsing at $76 \mathrm{MHz}$, the output voltage was pre-amplified, filtered by a low pass filter (BLP-21.4+, Mini-Circuits) and then terminated by a $50 \Omega$ resistor. The photodiode was connected to a radio frequency lock-in amplifier (SR844, Stanford Research Systems) referenced to the AOM driver. A $20 \mu$ s integration time was chosen and images generated by recording the $X$ output on the lock-in amplifier, and this resulted in a 12 second frame rate of $512 \times 512$ pixels images. Two-photon excited fluorescence (TPEF) signal was simultaneously detected in the epi-direction using a $805 \mathrm{~nm}$ dichroic mirror (DMSP805R, Thorlabs) and two filters centered at $660 \mathrm{~nm}$ (660.0 IF 40D, Ealing) to separate the signal from the laser fundamental and then detected by a photomultiplier tube (PMT, R3896, Hamamatsu). Image acquisition and processing were performed using Scanlmage (Vidrio Technologies) and Fiji (National Institute of Health, NIH, open source software), respectively. For the SRS, spectral acquisition regions of interest were acquired as the OPO was sequentially tuned to provide pump wavelengths, giving values of $\omega_{p}-\omega_{s}$ over the spectral range $2200-2250 \mathrm{~cm}^{-1}$. Intensity variations of the OPO signal were corrected by normalising each data point against the OPO signal intensity, which was recorded with a PIN photodiode. The linear power dependence of SRS signal on pump beam intensity allows compensation by straightforward linear normalisation.

\section{Spontaneous Raman Spectroscopy}

Spontaneous Raman spectra of the fludioxonil formulations were acquired using a Renishaw RM1000 Raman microscope (RENISHAW, Wotton-Under-Edge, UK) 
equipped with a 1200 lines $/ \mathrm{mm}$ grating providing a spectral resolution of $1 \mathrm{~cm}^{-1}$ and a diode laser providing excitation at $785 \mathrm{~nm}$ with up to $300 \mathrm{~mW}$ power. The system was calibrated prior to every spectral acquisition using the Raman band of a silicon wafer at $521 \mathrm{~cm}^{-1}$. Spectral data were acquired using Renishaw v.1.2 WiRE software.

\section{Seed Coating Preparation}

An aqueous suspension of fludioxonil particles stabilised by ionic and non-ionic surfactants, both with and without vegetable oil, was diluted into water, coated onto wheat seeds by dropping $\mathbf{0 . 1 2}$ gram diluted formulation onto $\mathbf{3 0}$ gram wheat seeds in a $60 \mathrm{ml}$ glass jar and shaking vigorously for 2 minutes, and allowed to dry at room temperature overnight. The wheat seed variety Savannah was used, and seeds were provided by Syngenta (Jealott's Hill International Research Centre, Bracknell, UK). To image the sliced wheat seed surfaces with forward-detected SRS signal, the appropriate sectioning direction (section parallel to the surface of the seed) was essential to minimise movement of the coating. Hand sectioning of wheat seeds was used to produce cross sections and transverse sections of the seed coat. These thin slices were mounted between two coverslips (intact wheat seeds were directly placed on a coverslip for epi-SRS detection) and imaged immediately.

\section{RESULTS AND DISCUSSION}

Figure $1 \mathrm{~b}$ shows the chemical structure of fludioxonil. It contains a cyano bond $(\mathrm{C} \equiv \mathrm{N})$ which provides a unique Raman peak that appears in the 'silent region' (2000 - 2700 $\mathrm{cm}^{-1}$ ) of the spectrum where there is no interference from endogenous seed components. We measured the spontaneous Raman and SRS spectra of fludioxonil formulated with and without vegetable oil. The spontaneous Raman spectrum of fludioxonil with oil shows strong peaks for the $\mathrm{CH}_{2}$ band $\left(2845 \mathrm{~cm}^{-1}\right)$ and the $\mathrm{C} \equiv \mathrm{N}$ band $\left(2219 \mathrm{~cm}^{-1}\right)$, indicating the presence of both lipid and fludioxonil. The result of the hyperspectral SRS scan is consistent with the spontaneous Raman spectrum. As expected, the spectrum of fludioxonil without vegetable oil shows lower intensity of $\mathrm{CH}$ stretching at $2845 \mathrm{~cm}^{-1}$, revealing that it has a lower lipid content. To image a specific active ingredient, on-resonance $\left(2219 \mathrm{~cm}^{-1}\right)$ and off-resonance $\left(2204 \mathrm{~cm}^{-1}\right)$ stimulated Raman imaging is typically performed to confirm the signal arises from stimulated Raman scattering, as shown in Figure 1c.

Figure $2 \mathrm{a}$ and Figure $2 \mathrm{~b}$ compares the $3 \mathrm{D}$ distribution of fludioxonil formulations on the surface of sectioned wheat seeds. False colours are assigned to individual Raman 
spectral frequencies to allow the chemically specific imaging of molecules. The formulation containing oil, Figure $2 \mathrm{~b}$, exhibits higher intensity of $\mathrm{CH}_{2}$ and $\mathrm{C} \equiv \mathrm{N}$ bands compared to that of the formulation without oil, Figure $2 \mathrm{a}$, which also has a less uniform distribution of fludioxonil. The coating performance of fungicide can be revealed by examining transverse sections (Figure $2 \mathrm{c}$ ), suggesting that fludioxonil is mainly concentrated on the surface of wheat seed coat.

A major drawback when performing forward-detected SRS microscopy is the need for sample sectioning prior to imaging since it could perturb the distribution of the seed coating. Although the use of cryo-sectioning would allow us to further immobilise the coating material than hand sectioning, the seed sectioning is still a destructive and time-consuming process ${ }^{[32]}$. We therefore performed imaging in the epi-detected SRS images with the introduction of quarter-wave plate and polarizing beam splitter in the epi-direction, as illustrated in Figure 3a. SRS microscopy equipped with the epi-mode detector offers an alternative way for surface studies on highly scattering, opaque, and thick tissues specimens ${ }^{[33-38]}$. In addition, TPEF of intrinsic molecules, such as chlorophyll and carotenoid, can be used as a good contrast for visualizing seed surface morphology (Figure $3 a)^{[31]}$.

To validate the multimodal performance of the epi-detection scheme, we acquired TPEF and SRS on-resonance $\left(2219 \mathrm{~cm}^{-1}\right)$ imaging of fludioxonil on the surface of intact wheat seed. As well as providing the spatial distribution of fungicide coating, the epi-SRS approach can also be used to acquire 3D images with the intrinsic optical sectioning capability of SRS (Figure 3b). As shown in the 3D reconstructed images, fludioxonil with oil could form a thin film on the coated seed surface as compared to without oil, suggesting that vegetable oil plays a significant role in seed coating performance. The SRS intensities at different depths of seed coats showed significant differences between the different formulations, as plotted in Figure $3 \mathrm{c}$.

Finally, we tracked the deeper coating performance of fungicides at a depth of $25 \mu \mathrm{m}$. The surface we defined in Figure 4 was the imaging plane where the SRS signal of CEN bond started to appear. We notice that those seeds coated by fludioxonil mixed with vegetable oil have more fungicide deposition and more even distribution than fludioxonil without oil (Figure $4 \mathrm{a}$ and Figure $4 \mathrm{~b}$ ). The coating area of fludioxonil increased from $409.6 \pm 81.9 \mu \mathrm{m}^{2}\left(2.5 \pm 0.5 \%\right.$ of total area) to $1507.3 \pm 393.2 \mu^{2}$ $(9.2 \pm 2.4 \%$ of total area), indicating a 3.7 -fold increase in deposition of seed coating fungicide (Figure 4c) mixed with oil, which is consistent with the increase of SRS signal strength when compared to the formulation without oil (Figure 4e). We further 
investigated the number of fludioxonil particles as a function of the coating area and found a rising trend of more coating areas with the usage of vegetable oil (Figure $4 d$ ). Based on the data in Figure 4c, Figure 4d and Figure 4e, our results demonstrate that the fungicide is deposited more efficiently on intact seed surfaces with the help of vegetable oil.

The results from this study demonstrate that a combined formulation of fludioxonil and vegetable oil would be more effective in coating superficial regions of the wheat seeds. There are several commercially available agrochemicals (such as azoxystrobin and chlorothalonil) also contain $\mathrm{C} \equiv \mathrm{N}$ groups, demonstrating the broad applicability of SRS microscopy as an analytical tool for the crop protection and agrochemical industry. To further improve this study, it would be possible to image multiple Als simultaneously on the surface of seeds by monitoring other useful Raman bands such as the deuterium (when using deuterium labelled materials) ${ }^{[39-41]}$, alkynes $(\mathrm{C} \equiv \mathrm{C})^{[42-44]}$, phenyl-diyne ${ }^{[45]}$, and carbon-halogen bond such as $\mathrm{C}-\mathrm{F}$ bond. Moreover, we can perform time-lapse imaging to reveal the quantitative, dynamic interaction between the seeds and Als.

\section{CONCLUSION}

In this study, we have demonstrated that SRS microscopy enables the distribution of Als to be mapped non-destructively on the surface of wheat seeds with high spatial-resolution without any external labelling. Our method can evaluate coating efficacy, revealing Al distribution on intact seeds at sub-micron resolution. To our knowledge, this is the first demonstration of in situ chemically specific mapping of fungicides in seed coats using nonlinear microscopy in a label free manner. We have shown that epi-SRS and TPEF microscopy with fibre laser excitation is an excellent tool for imaging fludioxonil on intact seed surfaces. This capability offers a new tool for designing more efficient seed treatments and other types of agrochemical formulation.

\section{Notes}

The authors declare no competing financial interest.

\section{ACKNOWLEDGMENTS}


We thank the funding from the Biotechnology and Biological Sciences Research Council (BBSRC, BB/M017915/1 and BB/K013602/1). We also would like to acknowledge the materials provided by Syngenta to undertake all research reported in this manuscript. 


\section{REFERENCES}

[1] K. K.Sharma, U. S. Singh, P. Sharma, A. Kumar, L. Sharma, J. Appl. Nat. Sci. 2015, 7, 521-539.

[2] E. Kadege, H. J. F. Lyimo, Archives of Phytopathology and Plant Protection 2015, 48, 601-610.

[3] S. R. Jacob, M. B. A. Kumar, E. Varghese, S. N. Sinha, Scientia Horticulturae 2016, 204, 116-122.

[4] R. Sur, A. Stork, Bulletin of Insectology 2003, 56, 35-40.

[5] B. Ivanova, M. Spiteller, Analyst 2012, 137, 3355-3364.

[6] A.-S. Jääskeläinen, U. Holopainen-Mantila, T. Tamminen, T. Vuorinen, Journal of Cereal Science 2013, 57, 543-550.

[7] C. Conti, M. Realini, C. Colombo, K. Sowoidnich, N. K. Afseth, M. Bertasa, A. Botteon, P. Matousek, Analytical Chemistry 2015, 87, 5810-5815.

[8] W. Devarrewaere, D. Foqué, U. Heimbach, D. Cantre, B. Nicolai, D. Nuyttens, P. Verboven, Environmental Science \& Technology 2015, 49, 7310-7318.

[9] P. Vermeulen, P. Flémal, O. Pigeon, P. Dardenne, J. Fernández Pierna, V. Baeten, Journal of Spectral Imaging 2017, 6, 1-7, DOI: 10.1255/jsi.2017.a1.

[10] Y. A. Salanenka, A. G. Taylor, Acta horticulturae 2008, 782, 151-154, DOI:

10.17660/ActaHortic.2008.782.16.

[11] C. W. Freudiger, W. Min, B. G. Saar, S. Lu, G. R. Holtom, C. He, J. C. Tsai, J. X. Kang, X. S. Xie, Science 2008, 322, 1857-1861.

[12] J.-X. Cheng, X. S. Xie, Science 2015, 350, aaa8870, DOI:10.1126/science.aaa8870.

[13] W. J. Tipping, M. Lee, A. Serrels, V. G. Brunton, A. N. Hulme, Chem. Soc. Rev. 2016, 45, 2075-2089.

[14] Y. Ozeki, W. Umemura, Y.Otsuka, S. Satoh, H. Hashimoto, K. Sumimura, N. Nishizawa, K. Fukui, K. Itoh, Nat. Photonics 2012, 6, 845-851.

[15] C.-S. Liao, P. Wang, P. Wang, J. Li, H. J. Lee, G. Eakins, J.-X. Cheng, Science Advances 2015, 1, e1500738, DOI: 10.1126/sciadv.1500738.

[16] C. W. Freudiger, W. Yang, G. R. Holtom, N. Peyghambarian, X. S. Xie, K. Q. Kieu, Nat. Photonics 2014, 8, 153-159.

[17] Z. Yu, T. Chen, X. Zhang, D. Fu, X. Liao, J. Shen, X. Liu, B. Zhang, X. S. Xie, X.-D. Su, J. Chen, Y. Huang, Chemical Science 2012, 3, 2646-2654.

[18] J. Moger, N. L. Garrett, D. Begley, L. Mihoreanu, A. Lalatsa, M. V. Lozano, M. Mazza, A. Schatzlein, I. Uchegbu, Journal of Raman Spectroscopy 2012, 43, 668-674.

[19] N. A. Belsey, N. L. Garrett, L. R. Contreras-Rojas, A. J. Pickup-Gerlaugh, G. J. Price, J. Moger, R. H. Guy, J. Controlled Release 2014, 174, 37-42. 
[20] D. A. Orringer, B. Pandian, Y. S. Niknafs, T. C. Hollon, J. Boyle, S. Lewis, M.

Garrard, S. L. Hervey-Jumper, H. J. L. Garton, C. O. Maher, J. A. Heth, O. Sagher, D. A.

Wilkinson, M. Snuderl, S. Venneti, S. H. Ramkissoon, K. A. McFadden, A.

Fisher-Hubbard, A. P. Lieberman, T. D. Johnson, X. S. Xie, J. K. Trautman, C. W.

Freudiger, S. Camelo-Piragua, Nat. Biomed. Eng. 2017, 1, 0027, DOI:

10.1038/s41551-016-0027.

[21] W. S. Chiu, N. A. Belsey, N. L. Garrett, J. Moger, M. B. Delgado-Charro, R. H. Guy, Proc. Natl. Acad. Sci. U. S. A. 2015, 112, 7725-7730.

[22] N. L. Garrett, B. Singh, A. Jones, J. Moger, J. Biomed. Opt. 2017, 22, 066003, DOI: 10.1117/1.JBO.22.6.066003.

[23] B. G. Saar, Y. Zeng, C. W. Freudiger, Y.-S. Liu, M. E. Himmel, X. S. Xie, S.-Y. Ding, Angewandte Chemie 2010, 122, 5608-5611.

[24] D. Fu, F.-K. Lu, X. Zhang, C. Freudiger, D. R. Pernik, G. Holtom, X. S. Xie, Journal of the American Chemical Society 2012, 134, 3623-3626.

[25] J. C. Mansfield, G. R. Littlejohn, M. P. Seymour, R. J. Lind, S. Perfect, J. Moger, Analytical Chemistry 2013, 85, 5055-5063.

[26] G. R. Littlejohn, J. C. Mansfield, J. T. Christmas, E. Witterick, M. D. Fricker, M. Grant, N. Smirnoff, R. Everson, J. Moger, J. Love, Front. Plant Sci. 2014, 5, 140.

[27] C.-C. Wang, D. Chandrappa, N. Smirnoff, J. Moger, Proc. SPIE 2015, 9329, 93291J. [28] B. Liu, P. Wang, J. I. Kim, D. Zhang, Y. Xia, C. Chapple, J.-X. Cheng, Analytical Chemistry 2015, 87, 9436-9442.

[29] G. R. Littlejohn, J. C. Mansfield, D. Parker, R. Lind, S. Perfect, M. Seymour, N. Smirnoff, J. Love, J. Moger, Plant Physiology 2015, 168, 18-28.

[30] Y. Wakisaka, Y. Suzuki, O. Iwata, A. Nakashima, T. Ito, M. Hirose, R. Domon, M. Sugawara, N. Tsumura, H. Watarai, T. Shimobaba, K. Suzuki, K. Goda, Y. Ozeki, Nat. Microbiology 2016, 1, 16124, DOI:10.1038/nmicrobiol.2016.124.

[31] C.-C. Wang, F.-Y. Yoong, S. Penfield, J. Moger, Proc. SPIE 2017, 10069, 1006928, DOI: $10.1117 / 12.2252080$.

[32] V. Converso, S. Fearn, E. Ware, D. S. McPhail, A. J. Flemming, J. G. Bundy, Scientific Reports 2017, 7, 10728.

[33] B. G. Saar, C. W. Freudiger, J. Reichman, C. M. Stanley, G. R. Holtom, X. S. Xie, Science 2010, 330, 1368-1370.

[34] M. N. Slipchenko, H. Chen, D. R. Ely, Y. Jung, M. T. Carvajal, J.-X. Cheng, Analyst 2010, 135, 2613-2619.

[35] P. Wang, M. N. Slipchenko, B. Zhou, R. Pinal, J. X. Cheng, IEEE Journal of Selected Topics in Quantum Electronics 2012, 18, 384-388.

[36] M. N. Slipchenko, R. A. Oglesbee, D. Zhang, W. Wu, J.-X. Cheng, Journal of Biophotonics 2012, 5, 801-807. 
[37] M. Ji, D. A. Orringer, C. W. Freudiger, S. Ramkissoon, X. Liu, D. Lau, A. J. Golby, I. Norton, M. Hayashi, N. Y. R. Agar, G. S. Young, C. Spino, S. Santagata, S.

Camelo-Piragua, K. L. Ligon, O. Sagher, X. S. Xie, Science Translational Medicine 2013, 5, 201ra119, DOI: 10.1126/scitransImed.3005954.

[38] F.-K. Lu, S. Basu, V. Igras, M. P. Hoang, M. Ji, D. Fu, G. R. Holtom, V. A. Neel, C. W. Freudiger, D. E. Fisher, X. S. Xie, Proc. Natl. Acad. Sci. U. S. A. 2015, 112, 1162411629.

[39] H. Yamakoshi, K. Dodo, A. Palonpon, J. Ando, K. Fujita, S. Kawata, M. Sodeoka, Journal of the American Chemical Society 2012, 134, 20681-20689.

[40] L. Wei, Y. Yu, Y. Shen, M. C. Wang, W. Min, Proc. Natl. Acad. Sci. U. S. A. 2013, 110, 11226-11231.

[41] L. Wei, F. Hu, Y. Shen, Z. Chen, Y. Yu, C.-C. Lin, M. C. Wang, W. Min, Nat. Methods 2014, 11, 410-412.

[42] A. Alfonso-García, S. G. Pfisterer, H. Riezman, E. Ikonen, E. O. Potma, J. Biomed. Opt. 2015, 21, 061003, DOI: 10.1117/1.JBO.21.6.061003.

[43] X. Chen, S. Grégoire, F. Formanek, J.-B. Galey, H. Rigneault, Journal of Controlled Release 2015, 200, 78-86.

[44] H. J. Lee, W. Zhang, D. Zhang, Y. Yang, B. Liu, E. L. Barker, K. K. Buhman, L. V. Slipchenko, M. Dai, J.-X. Cheng, Scientific Reports 2015, 5, 7930.

[45] W. J. Tipping, M. Lee, A. Serrels, V. G. Brunton, A. N. Hulme, Chemical Science 2017, 8, 5606-5615. 


\section{FIGURE LEGENDS}

FIGURE 1 Schematic of label-free microscopy and Raman spectra of fungicide. (a) Schematic of the optical setup and energy diagram. AOM: acousto-optic modulator, DM: dichroic mirror, OBJ: objective, FL: filter, PMT: photomultiplier tube, PD: photodiode, LIA: lock-in amplifier. (b) Left: Chemical structure of fludioxonil; Right: Spontaneous Raman (red and black solid lines) and SRS (blue circle) spectra of fludioxonil (contains a cyano bond, $\mathrm{C} \equiv \mathrm{N}$ ) mixed with and without vegetable oil. (c) Bright-field, on- and off-resonance SRS imaging of fungicide, fludioxonil, mixed with oil.

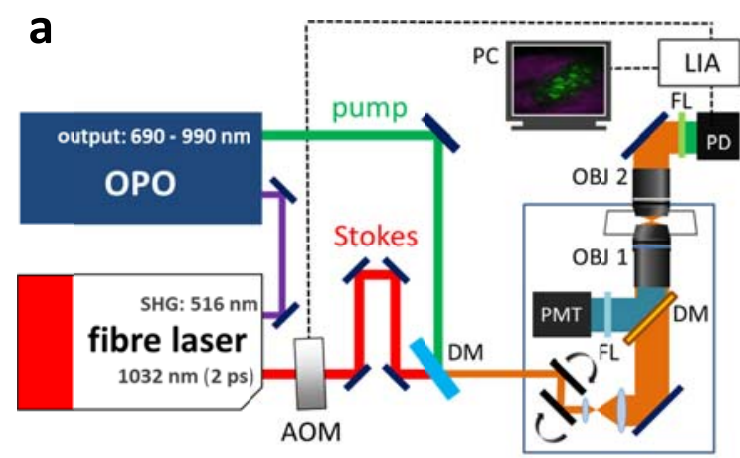

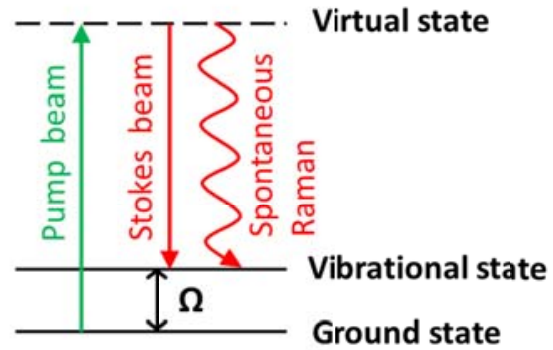

Energy diagram b<smiles>N#Cc1c[nH]cc1-c1cccc2c1OC(F)(F)O2</smiles>

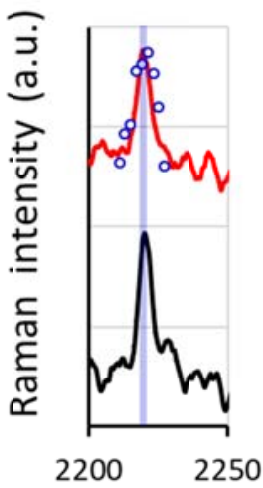

22502800

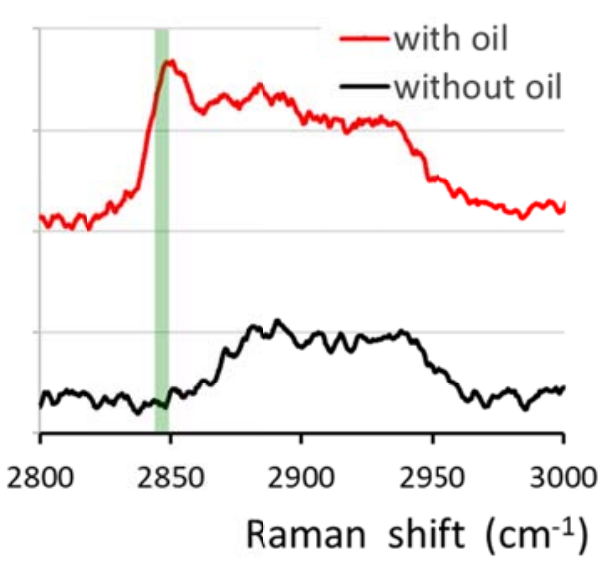

C

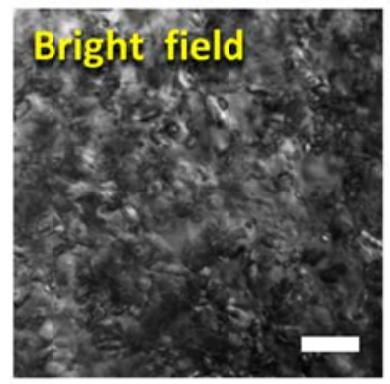

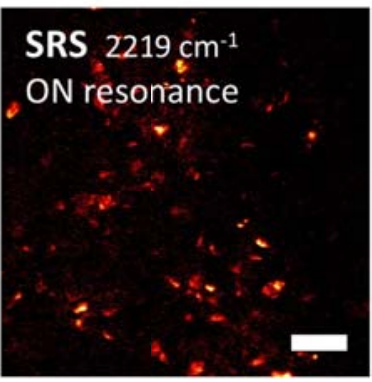

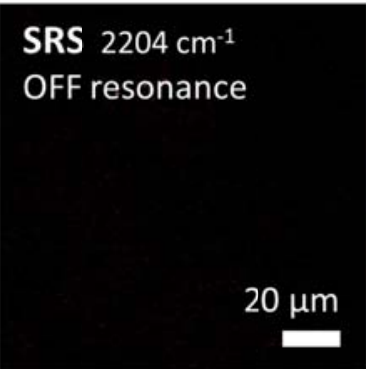

$\max$

$\min$ 
FIGURE 2 Chemically specific mapping of fungicide in seed coat. SRS imaging of $\mathrm{CH}_{2}$ $\left(2845 \mathrm{~cm}^{-1}\right)$ and $\mathrm{C} \equiv \mathrm{N}\left(2219 \mathrm{~cm}^{-1}\right)$ on the surface of wheat seeds as shown in (a) fludioxonil without oil and (b) fludioxonil with oil. (c) Distribution of fungicide with oil in the wheat seed transverse section.

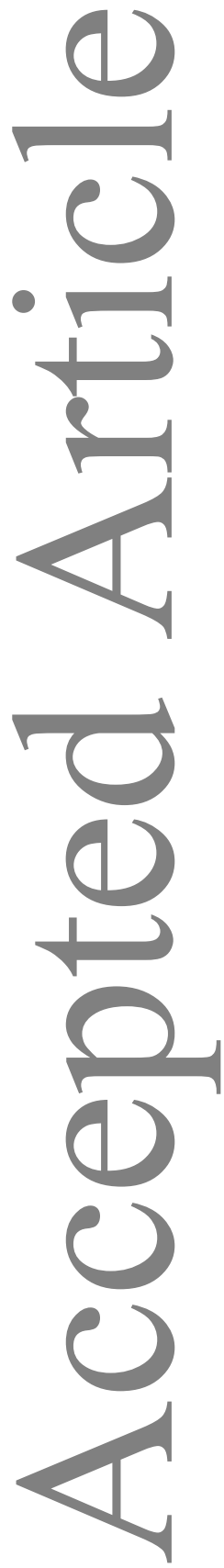

a
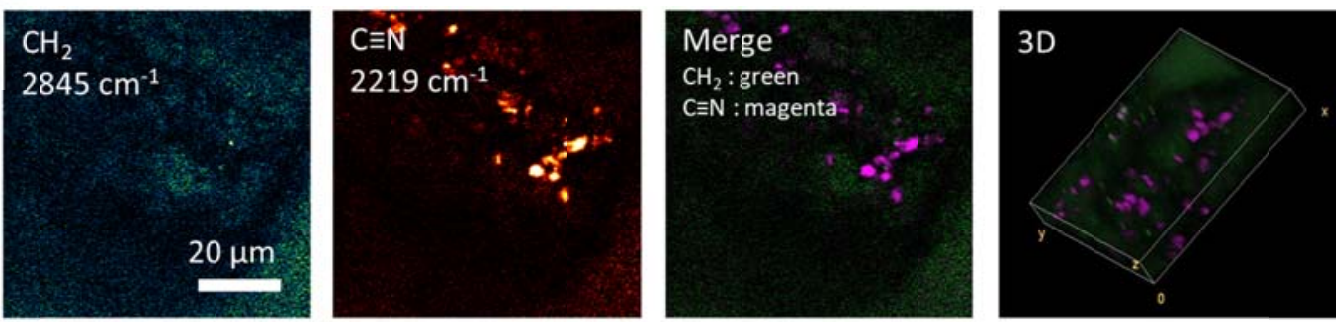

b
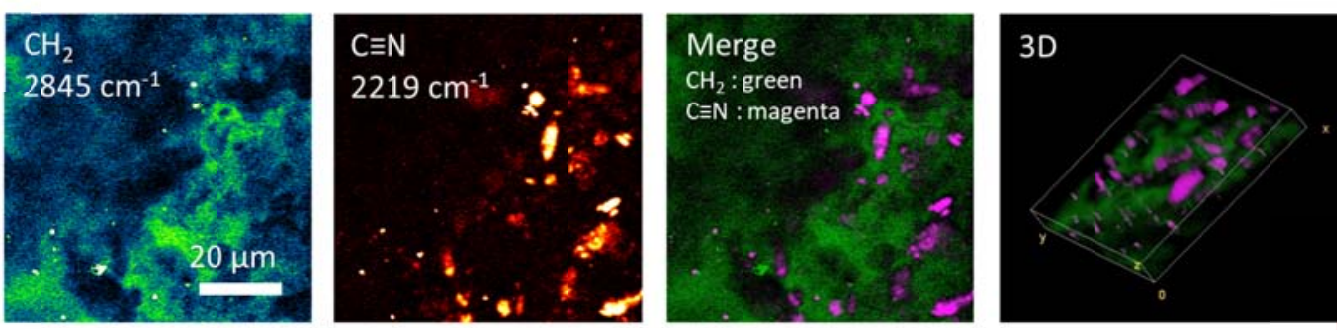

C
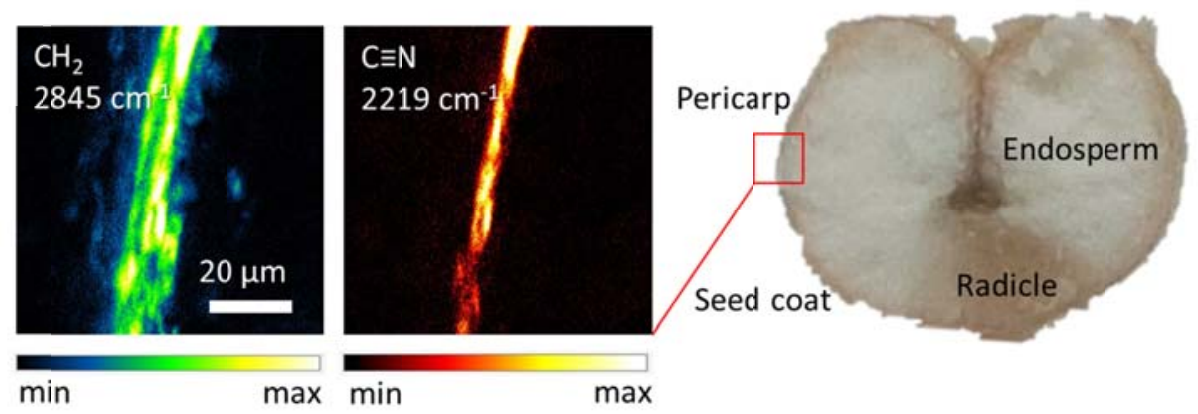
FIGURE 3 3D epi-SRS imaging of fungicide coated seeds. (a) Schematic of the epi-detected SRS and TPEF microscopy. DM: dichroic mirror, PBS: polarizing beamsplitter, QWP: quarter waveplate, OBJ: objective, FL: filter, PMT: photomultiplier tube, PD: photodiode, LIA: lock-in amplifier. (b) Comparison of the fludioxonil mixed with and without vegetable oil on the surface of wheat seeds. (c) Analysis of 3D distribution of fungicide coated wheat seeds.
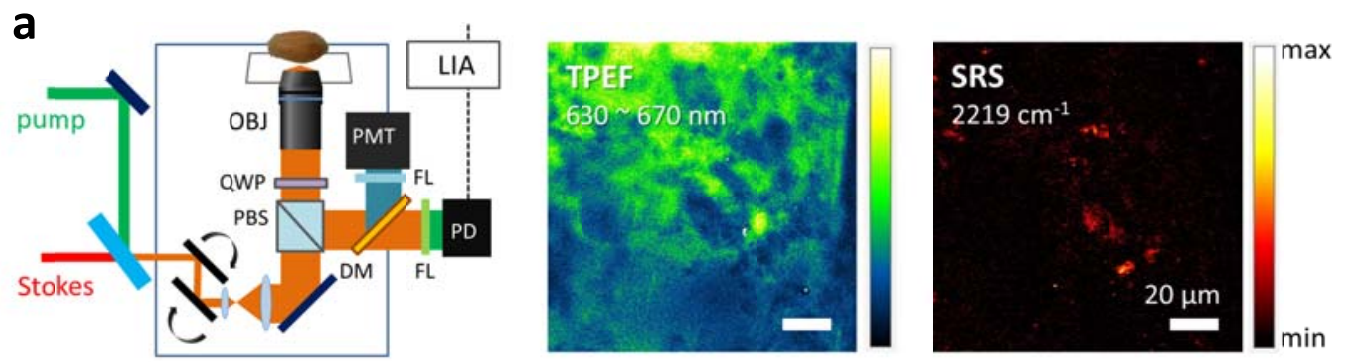

b

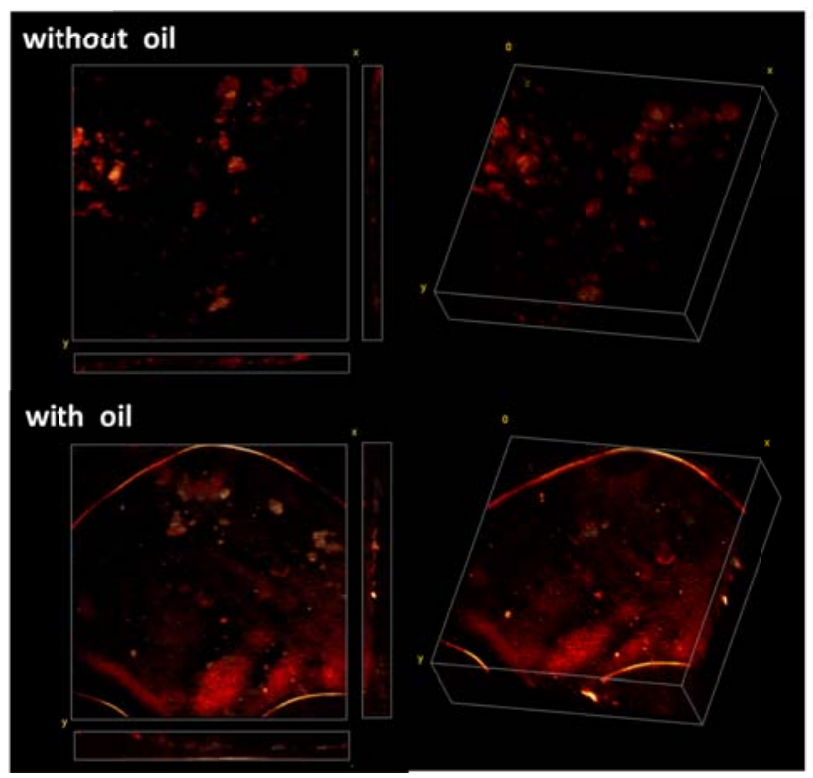

C

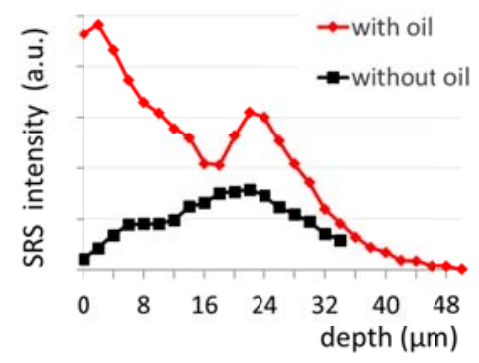


FIGURE 4 epi-SRS imaging of fungicide coated seeds at the depth of $25 \mu \mathrm{m}$. (a) Coating performance of fludioxonil mixed without vegetable oil on wheat seeds. (b) Coating performance of fludioxonil mixed with oil on wheat seeds. (c) Coating area percentage of fludioxonil mixed with oil compared to without oil. (d) Histogram of the number of fludioxonil particles as a function of the coating area. (e) Comparison of the SRS intensity of fludioxonil mixed with and without oil.

a
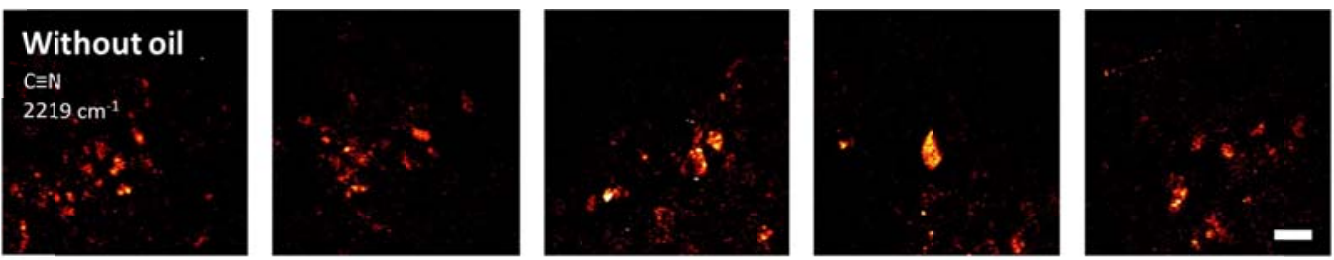

b
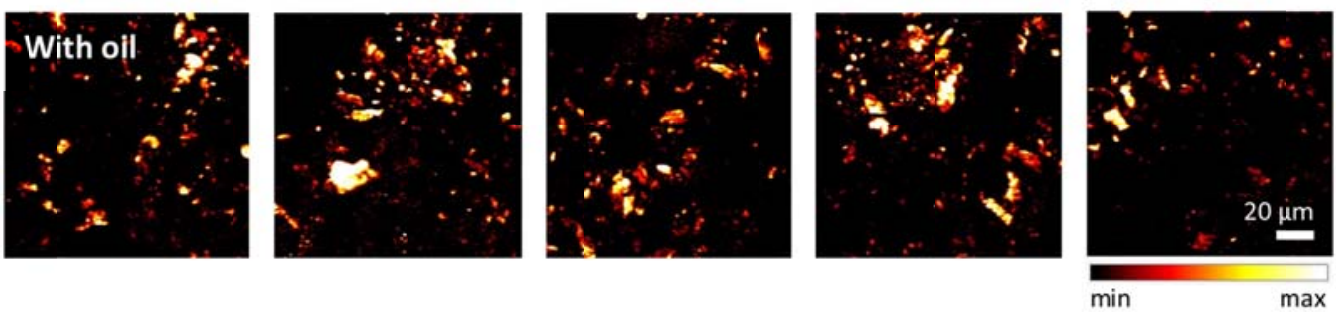

C

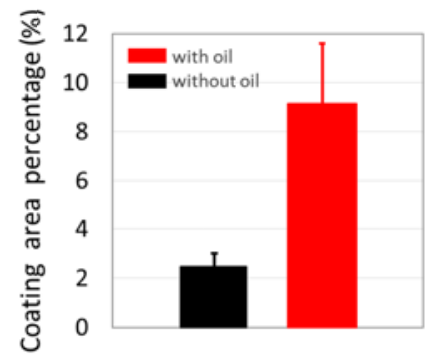

d

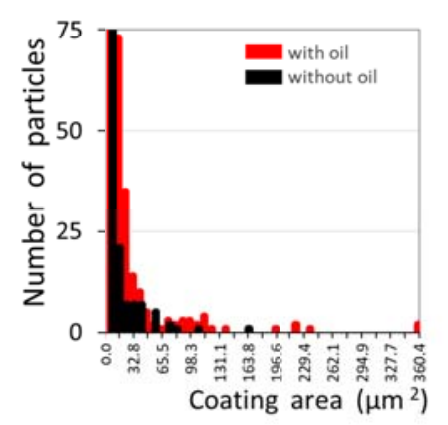

e

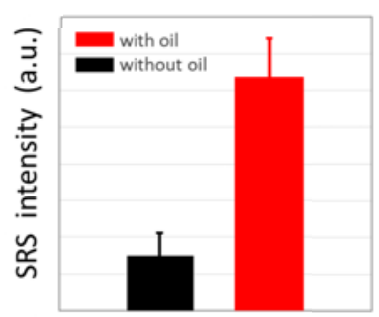




\section{Graphical Abstract}

In this work, we have demonstrated that SRS microscopy enables the distribution of active ingredient (Al) to be mapped non-destructively on the surface of vegetable seeds with high spatial-resolution without any external labelling. For the first time, in situ chemically specific mapping of seed coating has been performed, which provides novel insight that will lead to the rational engineering of more efficient seed treatments.

\section{TOC Figure}

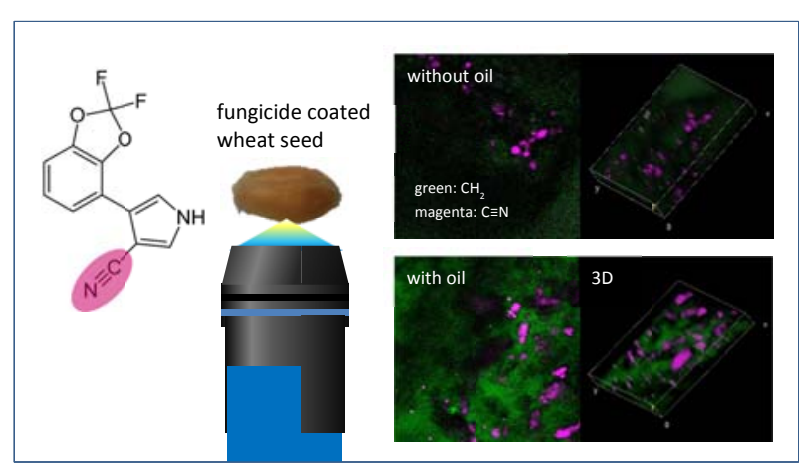

